\title{
$\infty \sqrt{1}$ Stroke and \\ Providing uninterrupted care during COVID-19 pandemic: experience from Beijing Tiantan Hospital
}

Xiaoyan Wang, ${ }^{1,2}$ Yu Chen (1) , ${ }^{1,2}$ Zixiao Li (D) , ${ }^{1,2}$ David Wang (D) ,
Yongjun Wang (D)

To cite: Wang X, Chen $Y, L i Z$, et al. Providing uninterrupted care during COVID-19 pandemic: experience from Beijing Tiantan Hospital. Stroke \& Vascular Neurology 2020;5: e000400. doi:10.1136/svn-2020-000400

Received 14 April 2020 Revised 4 May 2020

Accepted 5 May 2020

Published Online First 8 May 2020

Check for updates

(C) Author(s) (or their employer(s)) 2020. Re-use permitted under CC BY-NC. No commercial re-use. See rights and permissions. Published by BMJ.

${ }^{1}$ China National Clinical Research Center for Neurological Diseases, Beijing, China

${ }^{2}$ Beijing Tiantan Hospital, Capital Medical University, Beijing,

China

${ }^{3}$ Neurovascular Division, Department of Neurology, Barrow Neurological Institute, St. Joseph's Hospital and Medical Center, Phoenix, Arizona, USA

Correspondence to Dr Yongjun Wang; yongjunwang@ncrcnd.org.cn

\section{ABSTRACT}

Background The COVID-19 pandemic has already stressed the healthcare system in the world. Many hospitals have been overwhelmed by the large number of patients with COVID-19. Due to the shortage of equipment and personnel and the highly contagious nature of COVID-19, many other healthcare services are on hold. However, at Beijing Tiantan Hospital, a rapid response system has been in place so that routine care is not interrupted. We, therefore, would like to share our hospital-wide prevention and management policy during this pandemic to help other healthcare systems to function in this crisis.

Method Tiantan hospital is one of the leading neuroscience institutions in the world. With 1650 beds, its annual inpatient admission exceeds 30000 patients. Its COVID-19 rapid response policy was reviewed for its functionality.

Results There are nine key components of this policy: an incident management system; a comprehensive infection prevention and control, outpatient triage and flow system; a designated fever clinic; patient screening and administration; optimised surgical operations, enhanced nucleic acid testing; screening of returning employees; and a supervision and feedback system. In addition, a specific protocol was designed for treating patients with acute stroke.

Conclusion A comprehensive policy is helpful to protect the employee from infection and to provide quality and uninterrupted care to all who need these, including patients with acute ischaemic stroke.

Beijing Tiantan Hospital, Capital Medical University, is known for its outstanding medical care and groundbreaking research in neurology and neurosurgery. With 3210 full-time employees and 1650 inpatient beds, Beijing Tiantan Hospital is both a research hospital and a teaching hospital. Patient visits at Beijing Tiantan Hospital steadily increases over 1000000 outpatient visits and 30000 inpatient admissions per year.

The COVID-19 pandemic has already stressed the healthcare systems throughout the world. Rapid response to the outbreak of COVID-19 with timely prevention and management was implemented quickly at Beijing Tiantan Hospital. Healthcare services have not been interrupted for those who are critically ill, needing complex major surgeries, with high-risk pregnancy, tumours, stroke and haemodialysis. Here, we would like to share our experience on the policies implemented during this uncertain time.

\section{INCIDENT MANAGEMENT SYSTEM}

The Prevention and Control Leadership Group, chaired by the president of the hospital, was formed in Beijing Tiantan Hospital as the commanding centre for daily operations of COVID-19 prevention and management. A multidisciplinary planning committee was formed to facilitated the cooperation across all divisions, including the administration office, medical service office, office for disease control and prevention, infection management, nursing administration, outpatient clinic, pharmacy, hospital security, pharmaceutical equipment and materials management, bioengineering, general affairs, planning and construction division, and information and communications technology (figure 1). The 'Action Plan for Prevention and Control of COVID-19' was then developed. Daily meetings were scheduled for updates according to the local and national health guidance.

An ad hoc incident management group was formed. It included representatives from senior consultants, infection prevention and control, quality control and medical personnel. Medical consultation, education and training, and communication were conducted via either regular meetings or video teleconferences.

\section{COMPREHENSIVE INFECTION PREVENTION AND CONTROL}

Over 50 official policies have been released and updated by the prevention and control office on COVID-19 prevention and control for the emergency room and outpatient clinic, fever clinic, inpatient department, and 


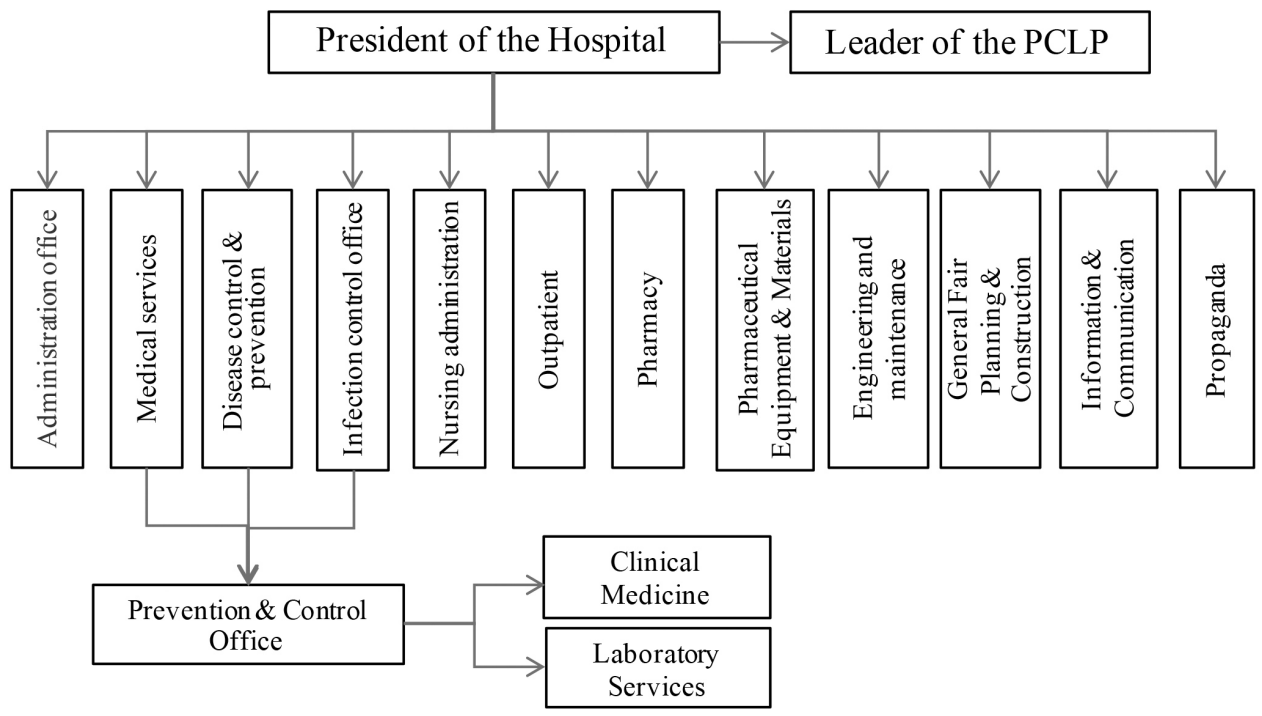

Figure 1 Beijing Tiantan Hospital incident management system. PCLP, Prevention and Control Leadership Group.

departments of surgery, supply, engineering and maintenance, environmental (housekeeping) services and human resources. Emergency preparedness plans were developed for the laboratory diagnosis centre, endoscope centre, operating rooms, ultrasound department, radiology department, pulmonary function examination and ECG rooms. A designated location, separated from other clinical areas, has been established for the admission of patients with possible COVID-19. A standard operating

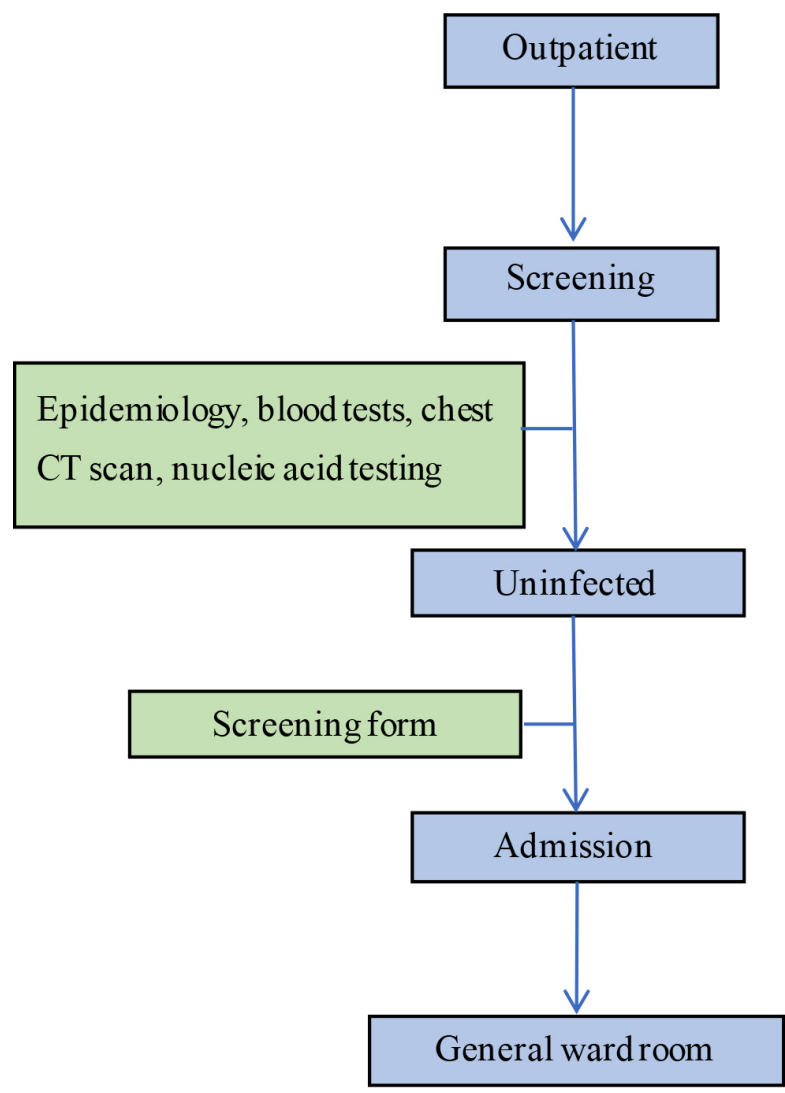

Figure 2 Flowchart of outpatient admissions. procedure and sterilisation process have been developed in all other departments.

\section{OUTPATIENT MANAGEMENT POLICY}

Clinical booking for doctor's appointment ensured the social distancing. All patients are required to make an appointment in the clinic, and for those urgent and lifethreatening cases, patients are advised to go to the emergency room. On checking in, a patient would have their body temperature checked, and questionnaires on any possible COVID-19 exposure and recent travelling history are filled out before going into a room. The respiratory clinic is now separated from other clinics. An isolation area has been set up in every clinic for patients with suspected COVID-19. All febrile patients will be sent to the fever clinic for further evaluation and therapy.

\section{FEVER CLINIC AS A VITAL PASS}

The fever clinic is located in the infection prevention and control building, which is separate from all other clinics. It is in charge of the screening, diagnosis and treatment of all febrile patients. Nucleic acid testing is used to confirm the diagnosis of COVID-19 when suspected based on physical signs and symptoms. The confirmed cases are then transferred to the COVID-19 designated hospitals. The patients who had been released from quarantine were required to undergo self-isolation and were followed up for further evaluation.

\section{PATIENT SCREENING AND ADMINISTRATION}

Emergency visits and admissions of patients for other medical conditions have decreased due to the epidemic. We designated a number of 340 reserved beds for COVID-19. Some units or services were combined in order to minimise the stuffing needs and to improve the 
operation efficiency. Each department designated three inpatient rooms for care of suspected COVID-19 cases that need ventilator support: one room for quarantine, next for buffering and the third one for sanitising.

A screening clinic has been set up since the outbreak of COVID-19 (figure 2). All patients admitted to the hospital from the outpatient clinic must be screened by a physician. These patients will have to fill out the questionnaires and have their body temperature taken, and undergo blood tests and a chest CT scan. The surgical patients must undergo further anaesthesia assessment before being admitted to the hospital. Nucleic acid testing for COVID-19 must be performed on all patients. Only patients who tested negative twice could be admitted.

All patients who come to the emergency room must be screened with a chest CT scan and a throat swab culture. According to their illness, they will be admitted to the transitional care unit (TCU) of the corresponding department if suspected of COVID-19 in order to reduce the risk of infection. These TCUs have been set up in the departments of neurosurgery, neurology, surgery, and internal medicine since 25 February 2020. Only patients who test negative twice in the TCUs could be admitted to the general ward rooms (figure 3).

\section{OPTIMISE ENDOVASCULAR TREATMENT FOR ACUTE STROKE AND SURGICAL OPERATIONS TO ENSURE SAFETY}

The management of the operating rooms and the interventional and surgical procedures have been adjusted to ensure endovascular treatment and surgical safety (figures 4 and 5). Outpatient operations are performed only after COVID-19 screening was negative. All operations on patients with suspected COVID-19 must be done in a negative pressure operating room with strictly controlled air flow. A protocol has been in place to facilitate the transport of suspected cases within the facility.

\section{ENHANCE NUCLEIC ACID TESTING}

Beijing Tiantan Hospital is one of the few hospitals in Beijing that could apply nucleic acid testing for COVID-19 during the initial phase of the epidemic. By performing nucleic acid testings to all febrile patients and admitted patients, the risk of nosocomial infection has been significantly reduced, which facilitates the normal operation of the hospital.

\section{SCREENING OF RETURNING EMPLOYEES}

All staff, including contractors, volunteers and medical students, returning back to Beijing must also be screened by a physician at the screening clinic, including completing the epidemiological questionnaires, having their body temperatures taken, and undergoing blood tests and a chest X-ray. Only the staff who were tested and confirmed to be uninfected could get back to work.

\section{SUPERVISION AND IMPROVEMENT}

Given the decreased patient visits and admissions in some clinics, a number of medical staff from these clinics were trained for the job of prevention and control of nosocomial infections. An inspection team has been organised to ensure compliance with policies and procedures in

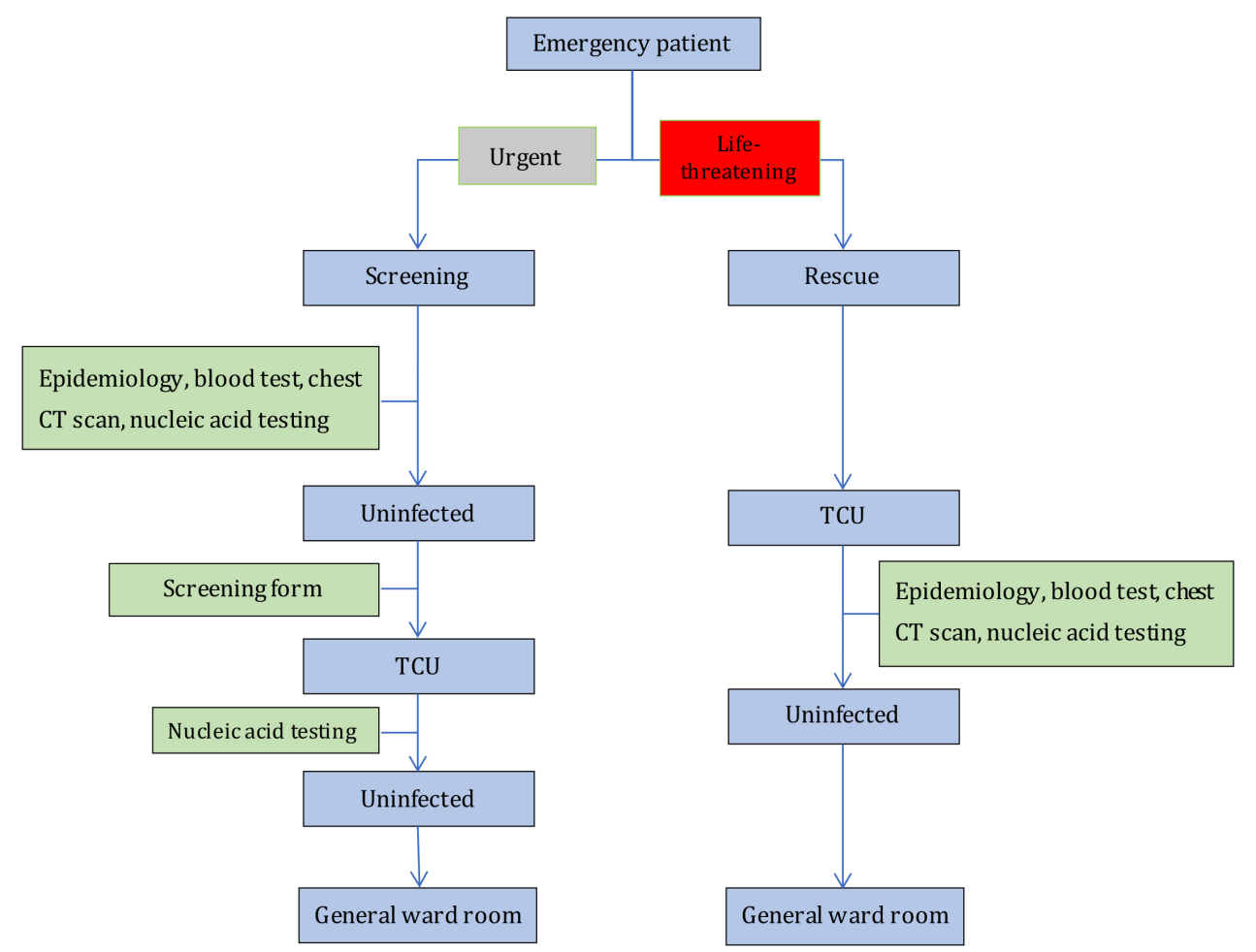

Figure 3 Flowchart of emergency admissions. TCU, transitional care unit. 


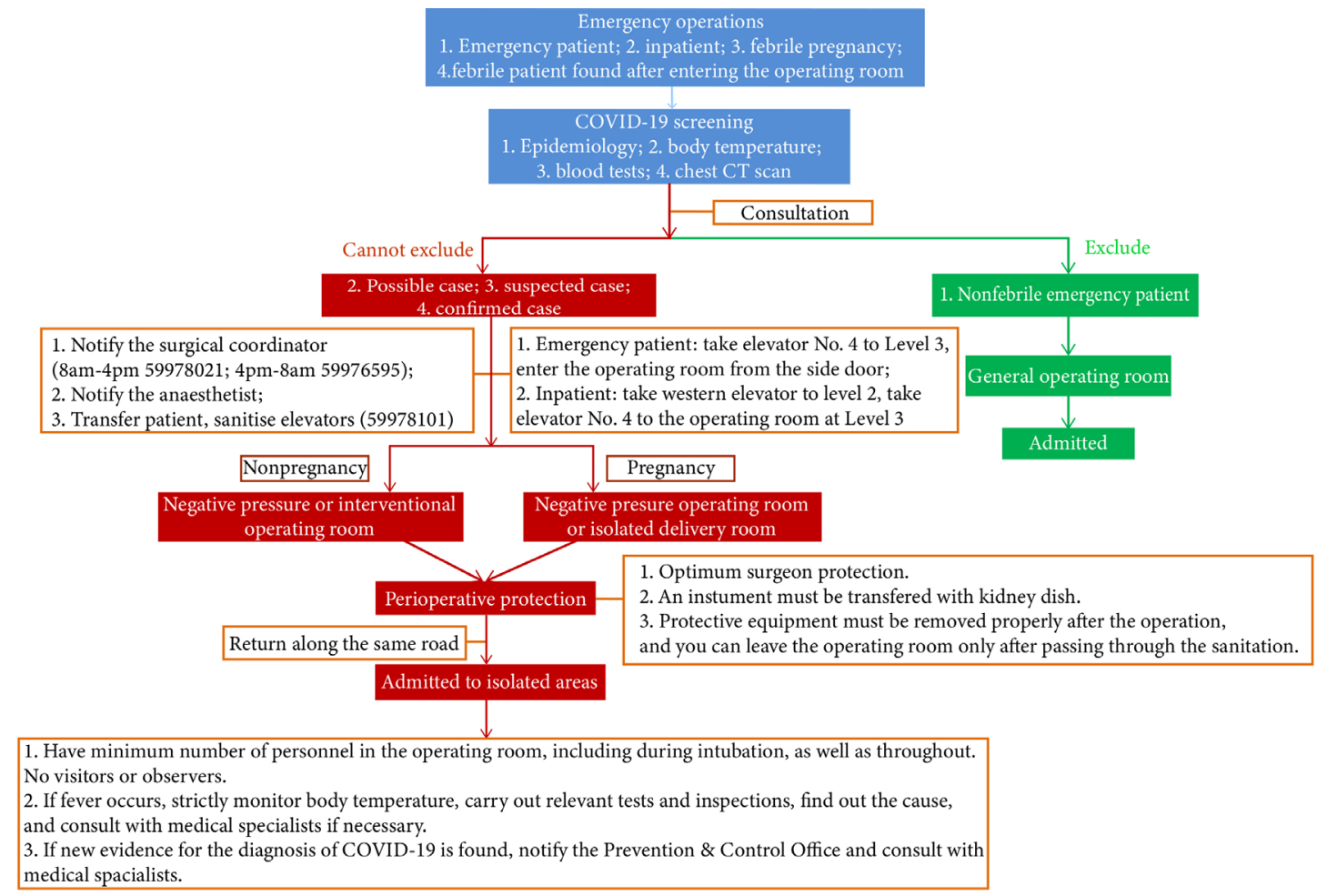

Types of emergency operations:

1. Nonfebrile patients: no epidemiological history; increased or decreased WBC count; atypical COVID-19 manifestations on chest CT; excluded by the consultation team.

2. Febrile patients: no epidemiological history; fever; increased or decreased WBC count;

atypical COVID-19 manifestations on chest CT; excluded by the consultation team.

3. Suspected cases: patients who meet the latest clinical diagnostic criteria of COVID-19

pneumonia; confirmed by teh consultation team; life-threatening and unable to be transferred.

4. Confirmed cases: patients who meet the latest clinical diagnostic criteria of COVID-19

pneumonia; confirmed by teh consultation team; nucleic acid testing positive; life-threatening

and unable to be transferred.

Figure 4 Flowchart of emergency operations. WBC, white blood cell.

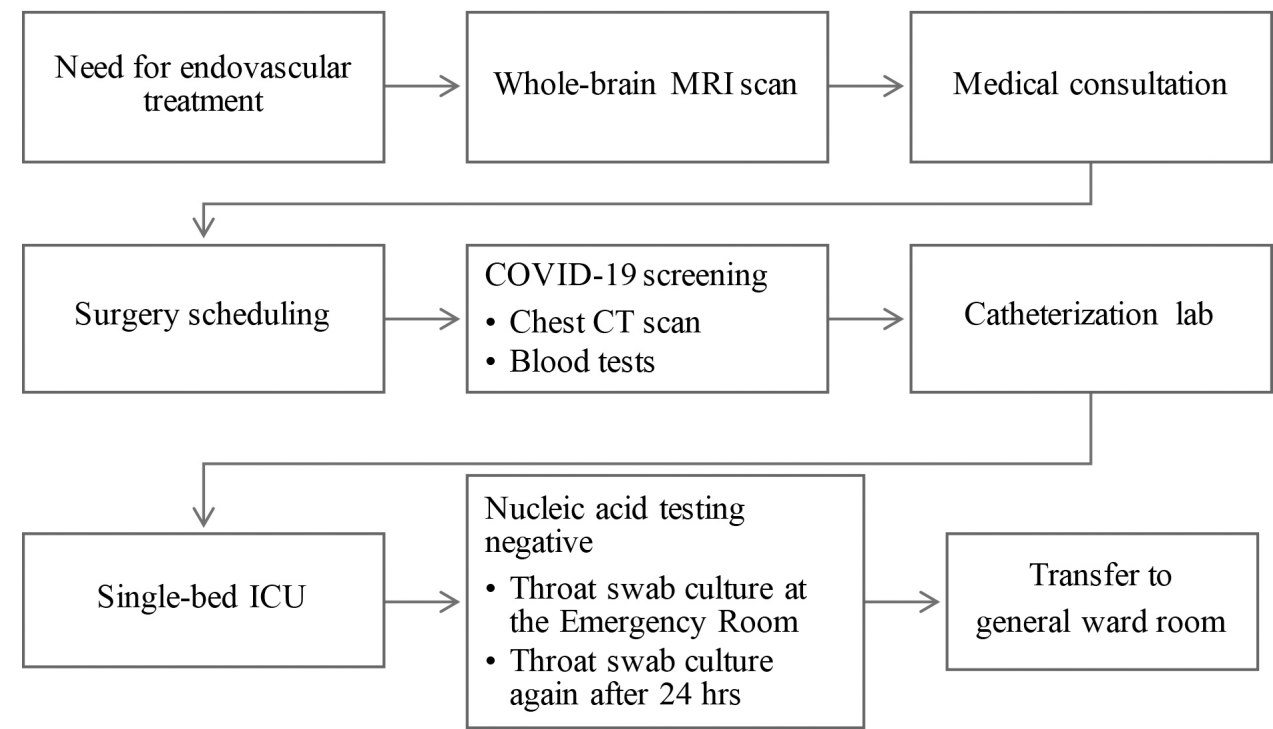

Figure 5 Flowchart of reperfusion therapy for acute ischaemic stroke. 
each clinic and department and to provide feedback to those that need improvement.

A timely, hospital-based response to manage the outbreak of COVID-19 plays a critical role within the health system in order to maintain services to the community. These preparation measures outlined in Beijing Tiantan Hospital might be helpful to other healthcare institutions that need similar policies in their fight against COVID-19.

Contributors YW and DW designed the concept. XW and YC wrote the paper. DW and ZL contributed to the conception, drafting and revision of the paper.

Funding This work was supported by Ministry of Science and Technology of the People's Republic of China (National Key R\&D Programme of China, GRANT NO: 2016YFC0901002, 2017YFC1310901, 2017YFC1307905, 2015BAl12B00).
Competing interests None declared.

Patient consent for publication Not required.

Provenance and peer review Not commissioned; externally peer reviewed.

Open access This is an open access article distributed in accordance with the Creative Commons Attribution Non Commercial (CC BY-NC 4.0) license, which permits others to distribute, remix, adapt, build upon this work non-commercially, and license their derivative works on different terms, provided the original work is properly cited, appropriate credit is given, any changes made indicated, and the use is non-commercial. See: http://creativecommons.org/licenses/by-nc/4.0/.

\section{ORCID iDs}

Yu Chen http://orcid.org/0000-0001-5863-7002

Zixiao Li http://orcid.org/0000-0002-4713-5418

David Wang http://orcid.org/0000-0003-2277-4608

Yongjun Wang http://orcid.org/0000-0002-9976-2341 